\title{
Research of Superfluorescent Fiber Source used in the Demodulation System of FBG Seismic Detector
}

\author{
Xiaobo Zhou ${ }^{1, a^{*}}$ and Shuangying $\mathrm{Jia}^{1, \mathrm{~b}}$ \\ ${ }^{1}$ Xijing university, Xi'an, Shaanxi, China \\ a369146317@qq.com, b925297483@qq.com
}

\begin{abstract}
Keywords: Superfluorescent Fiber Source(SFS); Seismic detector; Signal demodulation; Fiber Bragg Grating(FBG); Amplified Spontaneous Emission(ASE).
\end{abstract}

\begin{abstract}
Demodulation system for applied to seismic detection, erbium-doped fiber super fluorescent source combined with demodulation, the design of optical fiber Bragg seismic detection demodulation system based on ASE source, optical power output of $6.9 \mathrm{~mW}(8.43 \mathrm{dBm})$, spectral up along the width of $3 \mathrm{~nm}$ and down along the width of $2.5 \mathrm{~nm}$ range linear fitting are 0.9994 and 0.9995 respectively. The output spectrum of SFS has good linearity in the monotone interval, ensure the detection signal enough tuning range and good sensitivity, it was make the SFS can be reliably used for fiber Bragg grating seismic detector as edge filter demodulation method.
\end{abstract}

\section{Introduction}

Seismic exploration is the most mature and effective technology of geophysical exploration method, developed rapidly after world war II, it was one of a widely used methods on the exploration based on the artificial seismic reflection wave as the measured signal ,mainly used in oil, metals, coal, engineering geology, hydrogeology and geothermal exploration. Exploration technical requirements detector that can detect displacement, velocity and acceleration characteristics of seismic reflection and refraction signals, have a certain sensitivity and dynamic range as large as possible. By the quality of seismic data, determine the availability of good geological section and the correct geological explanation, and detector as the first part of field collection work, its importance is self-evident.

Seismic detector is a mechanical-electrical switching device that can change the mechanical vibration into electric signal, is a special vibration sensor applied to geological exploration and engineering survey. The seismic detector exist the problems: (1) the narrow band; (2) dynamic range is small; (3) the low accuracy and resolution; (4) poor anti-interference ability. Therefore, the research and exploration precision seismic detector and its demodulation technique theory are imminent.

The technology of optical fiber Bragg grating sensing has decades of development, with its high resolution, high signal-to-noise ratio and high fidelity, high definition, high accuracy, high reliability, provide the technological means for vibration measurement technology.[1,2] By using optical fiber Bragg grating as sensing element of detector, has a wide dynamic range, resistance to electromagnetic interference and signal bandwidth, easy to satisfy the demands of seismic exploration which high precision, high resolution.[3]

\section{Optical Fiber Bragg Seismic Detection Technology}

Basic Structure of Testing System for Optical Fiber Grating Vibration Sensor. Based on the research of FBG sensing property, the part of light which transmit to fiber-optics probe from the broadband light source, can be reflected within the fiber-optics probe of optical fiber Bragg grating when satisfy Bragg reflection conditions, and the change of the external vibration will cause the FBG reflection spectrum of center wavelength corresponding changes, then this part of the reflection spectrum that modulation of the external vibration information transmit to the sensor, the 
sensor processing the signal, and according to the need to convert it into signals can be observed in the secondary instrument $[4,5,6]$.

Principle of Demodulation System Based on SFS. As sensing element, FBG to expand its measuring range, wide spectrum light source be required; to improve the detection of signal to noise ratio, high power output of the light source should be asked. SFS is a kind of broadband light source of high stability, high power output [7], it can be used for demodulation system of fiber Bragg seismic detector.

The diagram of signal demodulation based on SFS and FBG as shown in Fig. 1. Pumped with the $980 \mathrm{~nm}$ laser diode, the ASE broadband light from a high concentrations of erbium-doped fiber, incident into the sensing FBG through the optical isolator and 3db coupler, the narrowband spectrum formed by sensing FBG reflection incident into photoelectric detector through $3 \mathrm{db}$ coupler and translate into the corresponding current signal, the current signal translate into voltage signal by current-to-voltage conversion circuit, through the low noise amplifier filter circuit and data acquisition system then entering the computer processing system, through a series of data processing can be measured results. The role of optical isolator is to make the light only one-way transmission, prevent that the light reflected by sensing FBG cannot spread into the SFS and influence its stability. By detecting the output voltage signal can speculate and calculate the wavelength of sensing FBG variation and achieve the goal of demodulation.

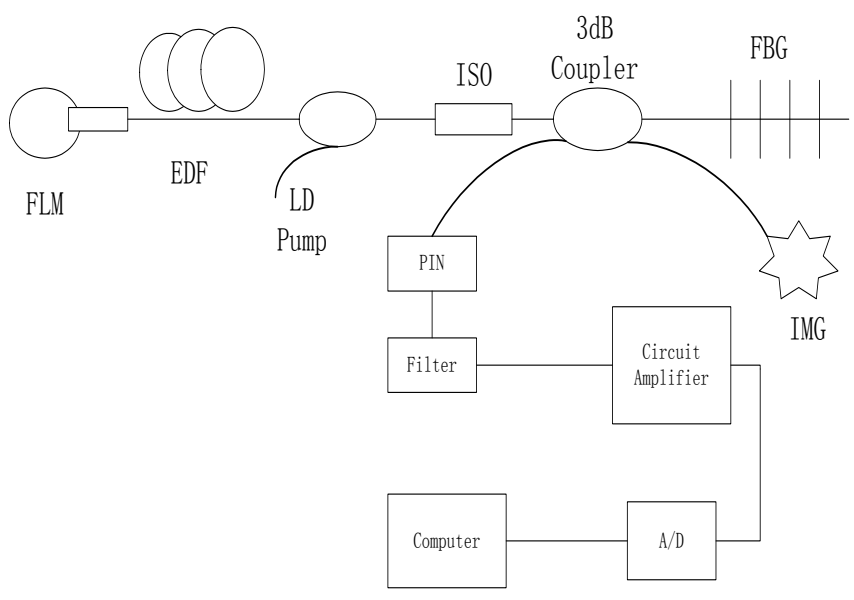

Figure 1. Signal demodulation based on ASE

\section{ASE Source Structure and the Result Analysis}

The optical power density that output from SFS does change with wavelength, within certain realms of wavelength ,the relationship between optical power density and wavelength is linear, the linearity essentially unchanged in a certain temperature range $[8,9,10]$, this feature can be used to realize demodulation.

For the demodulation system, wavelength resolution is mainly depend on slope of ASE source linear, minimum detectable power of photoelectric detection system and the signal-to-noise ratio of amplifying circuit are high and low. Therefore, in order to improve performance choice the parts of SFS that linear region is bigger and linearity is better. Linearity is higher, the greater the change of signal detection, and the higher the sensitivity; At the same time, high sensitivity is makes easy to signal detection, but also easy to detected unnecessary extra signal, therefore, also restrict certain limits of detection, so the demodulation system needs SFS provides light should not only have high linearity but also have larger linear range.

As shown in Fig. 1,in order to obtain high output optical power, SFS in demodulation system adopts double pass backward (DPB) configuration, use $980 \mathrm{~nm}$ laser diode for pumping source, the concentration of the erbium-doped fiber is greater than 1900 PPM, fiber numerical aperture(NA) is 
0.20 , the mode field diameter is $6.8^{\mu m}$, the cutoff wavelength is $960 \mathrm{~nm}, 980 \mathrm{~nm}$ peak absorption is $8.6 \mathrm{~dB} / \mathrm{m}$, near $1530 \mathrm{~nm}$ peak absorption is $14.2 \mathrm{~dB} / \mathrm{m}$, stretches 7 meters, in order to make the monotone interval of light output spectrum has a good linear.

Output spectral density of SFS as shown in Fig. 2, the optical output power is $6.9 \mathrm{~mW}$ $(8.43 \mathrm{dBm})$, can satisfy the use requirement of demodulation system. As seen in Fig. 2, the output spectrum has two monotone intervals, if the center wavelength of FBG in monotone interval, the FBG reflected light intensity is changing in a linear fashion with the wavelength change. The spectrum of ASE source respectively along the rising edge $1525.4 \mathrm{~nm} \sim 1528.4 \mathrm{~nm}$ and falling edge $1532 \mathrm{~nm} \sim 1534.5 \mathrm{~nm}$ for linear fitting.

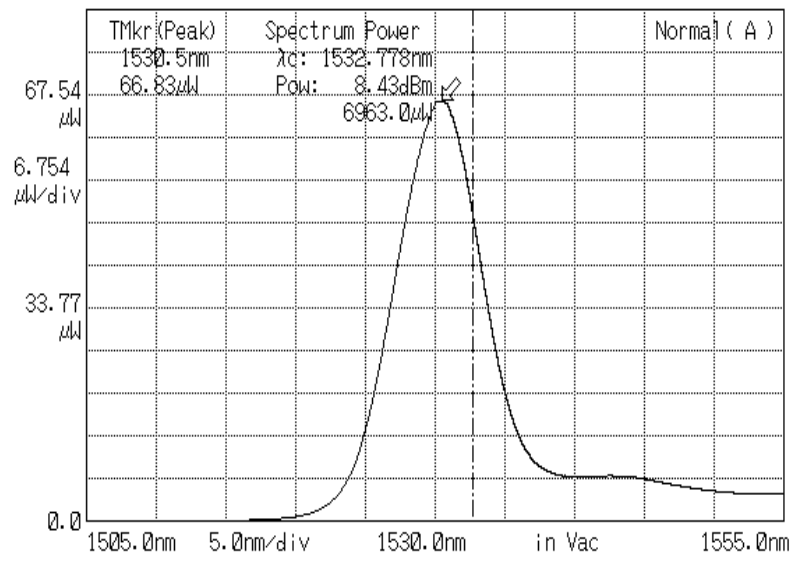

Figure 2. SFS spectral density figure in demodulation

By fitting curve, as seen that rising along the width of $3 \mathrm{~nm}$ range linear fitting degree is 0.9994 , falling edge width of $2.5 \mathrm{~nm}$ range linear fitting degree is 0.9995 , good linearity can ensure the detection signal has a good sensitivity, and linear bandwidth is large enough to ensure the detection signal can have enough tuning range. Fitting show that the edge of two flat sides can be used as the bevel edge of filter for signal demodulation.

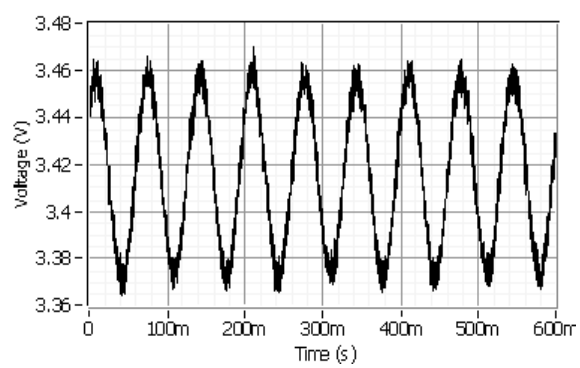

(a) $15 \mathrm{~Hz}$ direct measurement

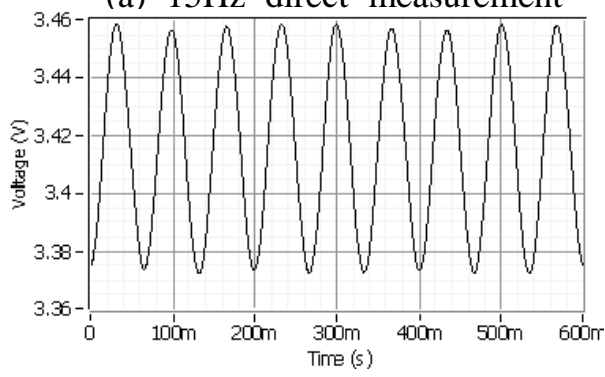

(c)15Hzsoftware restore signal

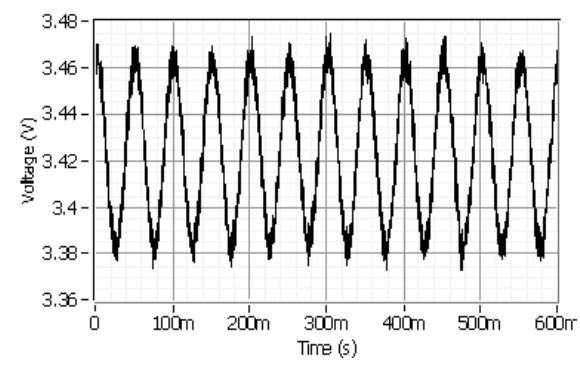

(b) $20 \mathrm{~Hz}$ direct measurement

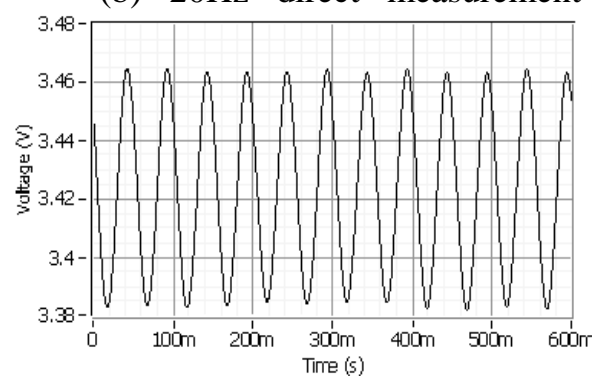

(d) $20 \mathrm{~Hz}$ software restore signal

Figure 3. Detection light intensity of voltage and sampling time 
The demodulation system is shown in Fig. 1, use the Bragg centre wavelength is about $1532 \mathrm{~nm}$ grating as sensing FBG, when ASE source illuminate the sensing FBG, its light intensity reflection cyclical change with it, through photoelectric receiver and amplifier module, obtain the voltage signal corresponding to strain, so as to achieve the purpose of dynamic strain measurement. Frequency characteristics of the test system mainly depend on the frequency characteristics of the speaker. The experiment, detect two vibration signals frequency of $15 \mathrm{~Hz}$ and $20 \mathrm{~Hz}$, electric signal of the photoelectric transformation output, sampling by data acquisition card with sampling frequency of $10 \mathrm{kHz}$, waveform as shown in Fig. 3,Fig. (a) (b) respectively direct measurement the voltage signal under $15 \mathrm{~Hz}$ and $20 \mathrm{~Hz}$ frequency, Fig. (c) (d) respectively reduction of signal after filtering software under $15 \mathrm{~Hz}$ and $20 \mathrm{~Hz}$ frequency. Can be seen from the figure, the detected voltage signal is good sine signal, suggesting that use of ASE source as edge filter demodulation method for fiber Bragg grating seismic detector is reliably.

\section{Summary}

Demodulation system for applied to seismic detection, erbium-doped fiber super fluorescent fiber source combined with demodulation, the design of optical fiber Bragg seismic detection demodulation system based on SFS, the output spectrum of SFS has good linear in the monotone interval, it was make the SFS can be used for fiber Bragg grating seismic detector as edge filter demodulation method.

\section{Acknowledgement}

Shaanxi Department of Education Special Research Project (2013JK1121);Xijing University Scientific Research Foundation Project(XJ120235); Xijing University Scientific Research Foundation Project(XJ140236).

\section{References}

[1] Y.B.Liao:Fibre Optical(Tsinghua University Press,China 2000).

[2] Y.J.Rao,Y.P.Wang and T.Zhu:The principle and application of fiber Bragg grating (Science Press ,China 2006 ).

[3] F.X. ZHANG, X.B.WU, S.J.LI, X.H.LIU, J.S.LV and J.LIU: Progress in Geophysics, 2014, Vol. 29(2014) No.5, p. 2456.

[4] X.Liu:Seismic geophone test method research (MS., Xi'an Shiyou University, China 2014) p.7.

[5] C.Ma,X.G.Qiao andZ.A.Jia: Progress in Geophys(in Chinese),Vol.23(2008)No.2,p.622.

[6] M.Shao, X.G.Qiao and D.Q.Feng:Journal of Optoelectronics Laser(in Chinese), Vol. 23 (2012) No.3, p. 418.

[7] M.Shao,X.G.Qiao and H.W.Fu:Progress in Geophys(in Chinese),Vol.26(2011)No.1,p.342.

[8] X.D.Ying:Study on Demodulation Technology for Fiber Bragg Grating Seismic Detection(MS., Xi' an Shiyou University, China 2011) p.29.

[9] Y.Yu,Z.H.Yu and L.J.Zhao:Transducer and Microsystem Technologies（in Chinese）, VOL. 30 (2011) No.6, p. 11.

[10]L.Li: Study on Erbium-doped Fiber Superfluorescence Source and the Gain Flattening Techniqe(MS., Xi'an ShiyouUniversity, China 2010) p.23. 